\title{
Detection of breast cancer using non-invasive $X$-ray diffraction technique of hair: A preliminary study
}

\author{
A. Maziar ${ }^{1}$, D. Shahbazi-Gahrouei1 ${ }^{*}$, M.B. Tavakoli ${ }^{1}$, V. Changizi ${ }^{2}$, \\ Z. Ghasemian'
}

${ }^{1}$ Department of Medical Physics, School of Medicine, Isfahan University of Medical Sciences, Isfahan, Iran

${ }^{2}$ Department of Medical Physics, Tehran University of Medical Sciences, Tehran, Iran

\section{Technical note}

\section{*Corresponding author:}

Dr. Daryoush Shahbazi-Gahrouei,

Fax: +983137929032

E-mail: shahbazi@med.mui.ac.ir

Revised: Feb. 2015

Accepted: March 2015

Int. J. Radiat. Res., April 2016; 14(2): 153-158

DOI: 10.18869 /acadpub.ijrr.14.2.153

\section{ABSTRACT}

Background: An early diagnosis of breast cancer relates directly to an accurate treatment plan and strategy. Early detection of breast cancer before its development would be a significant reduction of morbidity and mortality rates. The aim of this preliminary study is to investigate the sensitivity of Wide Angle X-ray diffraction (WAXRD) method on women hair samples of healthy and breast cancer patients in comparison with other modalities such as synchrotron based XRD beam and mammography. Materials and Methods: Hair samples were taken from occipital region of skull from healthy and breast cancer patients (43 women) were analyzed using X-ray diffraction and the results were analyzed and compared with mammography and pathology reports. Results: The results of analyzed samples showed the sensitivity for purposed WAXRD method was $86 \%$ in comparison with synchrotron based XRD beam (64\%) and also with mammography (70\%). Conclusion: This non-invasive method is less harmful and is more sensitive than the two other methods and help the physicians for choosing accurate treatment plan.

Keywords: X-ray diffraction (XRD), breast cancer, hair, detection, non-invasive.

\section{INTRODUCTION}

Early diagnosis of breast cancer is important for making decision on proper treatment planning. Because of high and also increasing worldwide morbidity and mortality rates of breast cancer (1-3), there are many researchers who interested in diagnosis and treatment of this type of neoplasm. Since the cure rate of cancer is highly dependent on stage of the disease, early detection of disorder is an important subject to choose an effective treatment strategy. To obtain an accurate and also proper treatment plan and strategy, there is strong need for reliable and also early diagnosis methods. The usual methods for detection of breast cancer after physical examination are mammography; which is gold standard, magnetic resonance imaging (MRI) and ultrasound $(3,4)$. Since detection of dense breasts is difficult in mammography(5), the radiographer and also the physician should be proficient (6). The sensitivity for mammography is about 70 percent, and for definite diagnosis of cancer there is a need of pathology and biopsy confirmation. It seems that a simple, less dangerous and safe method with high sensitivity could be initiated and implemented.

There are many methods for studying human tissue as an indicator of breast cancer such as molecular structure, protein crystallography or biomarkers evaluation $(7,8)$. Diffraction effect produces a characteristic scattering pattern of the tissue which has been irradiated. This signature is dependent upon the molecular composition of the target and hence could be used to characterize the tissue ${ }^{(8-10)}$.

James et al. (11) presented a method using 
synchrotron based X-Ray diffraction (XRD) which produced diffraction patterns of hair and showed a good correlation between healthy state of hair samples and related pattern. Saengkaew et al. (12) presented an analysis of human-hair microstructures by wide-angle Xray diffractions (WAXRD) and small-angle X-ray scattering (SAXS). In another study, Corino et al. (13) made a research with a special sample holder and get the related results between molecular structure of hair and presence of disease. They reported that synchrotron-derived X-ray diffraction has the potential ability to provide a non-invasive method to show the presence of breast cancer (13).

Several studies by other researchers confirmed an association between the XRD hair patterns and the presence of breast cancer (14). Limitations including of the complexity of interpretation the data and lack of synchrotron based on X-ray diffraction apparatus in all medical experiments centers, caused evaluation of XRD patterns using a facility other than synchrotron. For this reason, investigation of changes in diffraction pattern of hair samples of healthy and breast cancer patients using Wide Angle X-ray Diffraction (WAXRD) was proposed. At the end, advantages and disadvantages of the purposed method against previous synchrotron based method and mammography was investigated.

\section{MATERIALS AND METHODS}

In this study, hair samples of 43 women (including 14 healthy, 17 patient and 12 suspicious) individuals in four cancer treatment centers of Tehran hospitals were used. Healthy individuals were women those physical examinations and mammography reported as healthy, and cancer cases were those patients that had positive physical examination, mammography and also pathology reports. The suspicious individuals whom mammography reports were reported normal but the clinical evidences and examinations were suspicious.

All the sample donors were Iranian with Arian origin and with the age between 33-75 years old with average of $51.3 \pm 11.5$ years. The hair samples obtained from women who had no colored hair for 6 weeks or more and hair samples of cancer cases were collected from patients who had not delivered chemotherapy and medicine therapy. Hairs were cut from occipital region of skull and taking through sample preparation method introduced by the International Atomic Energy Association (IAEA) (15). In order to cleaning of samples from any kind of external contamination, coded samples were taking washing method with ethanol and distilled water for three times and then dried in oven about 30 minutes at $80{ }^{\circ} \mathrm{C}$ temperature. Then, the samples were cut with surgical stainless steel scissors into fine pieces about 2 $\mathrm{mm}$ and using a mortar the samples became powdered and drying in oven for about 10 hours at an $80{ }^{\circ} \mathrm{C}$ temperature (16).

Wide Angle X-Ray Diffraction (WAXRD) was used as measurement method. The procedure of sample preparation was as follow. The amount of $0.4 \mathrm{~g}$ of each powdered sample filled in special sample holders of XRD measuring system with $10 \times 10 \mathrm{~mm}^{2}$ exposure window and measured with X' Pert Pro MPD (PANalytical Inc., Netherlands) XRD system with copper anode in $40 \mathrm{kV}$ and $40 \mathrm{~mA}$ at $25{ }^{\circ} \mathrm{C}$ temperature. The detector was high resolution Germanium (Ge) semiconductor PIXcel (PANalytical Inc., Netherlands) detector. Starting and ending positions of each exposure were 2.02 and 89.97 degrees, respectively with $0.02^{\circ}$ step size and 48 seconds step scan time. The proportional curves in intensity (counts) versus $2 \theta$ (exposure angle) were drawn and studied.

The WAXRD results of coded hair samples were analyzed by XRD experts. These results were matched with donors' health state data. The results of healthy and cancer patient groups were compared with mammography and also pathology reports and based on the results, the sensitivity of the method versus mammography were also evaluated.

XRD curve of healthy and also breast cancer patients were proceed and statistics parameters such as mean and standard deviation values of peak heights (counts) and peak positions $(\theta)$ were analyzed with IBM SPSS (version 22.0, 
2013) software.

To show normal distribution of data, Shapiro-Wilk test was used. Students' $t$-test and non-parametric statistical tests were used to compare the data. To check the assumption of correlation data were used Pearson correlation coefficient was used to show correlation of data. $P$ value $<0.05$ was considered as significant level.

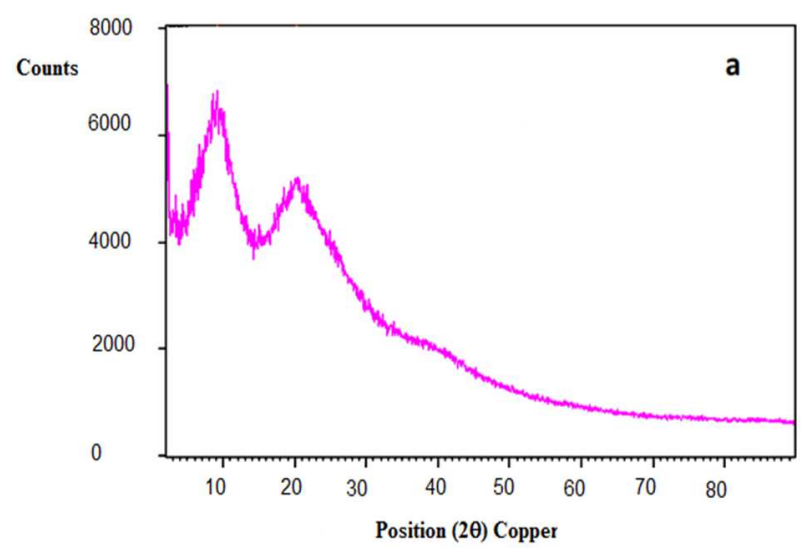

An example of X-ray diffraction pattern of healthy woman hairs and hairs from patient with breast cancer is shown in figure 1. As this figure shows, two distinguishable peaks with different heights (counts) are presented. Also, diffraction pattern of normal human hair and hair from a patient with breast cancer using synchrotron beam XRD method is shown in figure 2 .

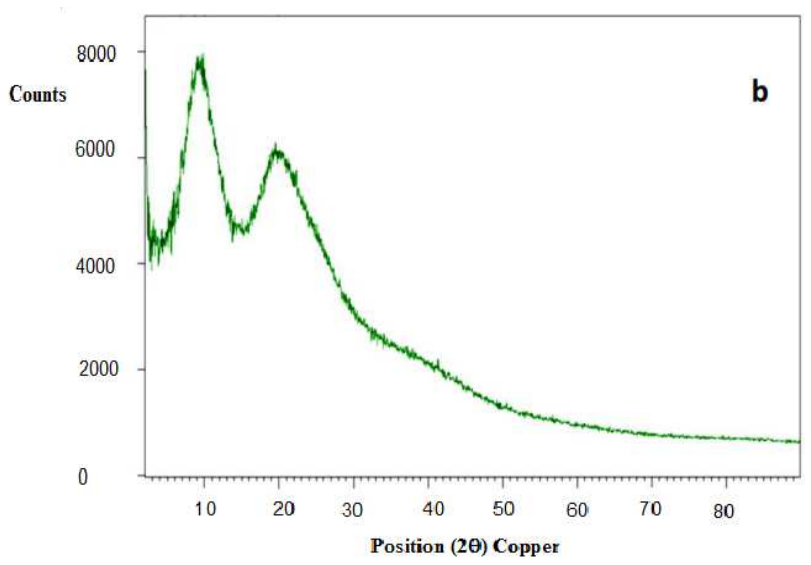

Figure 1. Examples of X-ray diffraction pattern of healthy human hairs (a) and hairs from patient with breast cancer (b) which showing two distinguishable peaks but in different heights (counts).

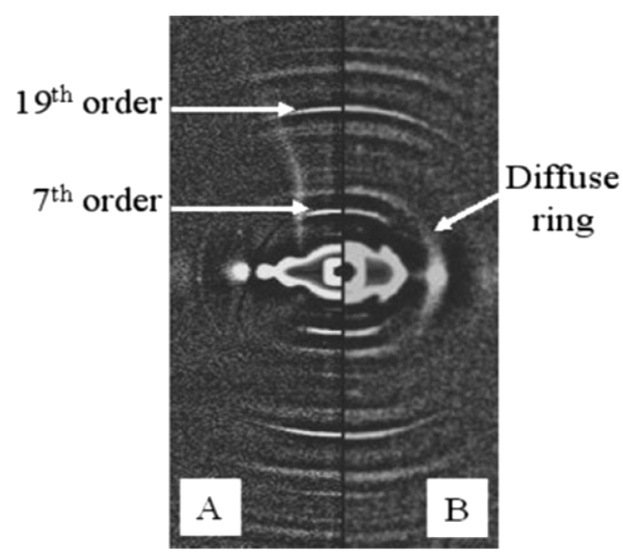

Figure 2. Diffraction pattern of (A) normal human hair and (B) hair from a patient with breast cancer using synchrotron beam $X R D$. The diffuse ring that has shown in $(B)$ indicates the presence of tumor.

\section{RESULTS}

Results of WAXRD experiment of hair samples belong to healthy and breast cancer patients are shown in figure 3 . As it seen, $1^{\text {st }}$ and $2^{\text {nd }}$ peaks height of breast cancer patients which are shown in counts (height) is more than counts related to healthy samples. So, X-ray diffraction patterns of hair from breast cancer patients have more intensity than that of normal.

Figure 3 also demonstrates the comparison between healthy and cancer patients. In addition, in each group, the results were compared with mammography and also pathology report of each individual and they are shown in table 1. 

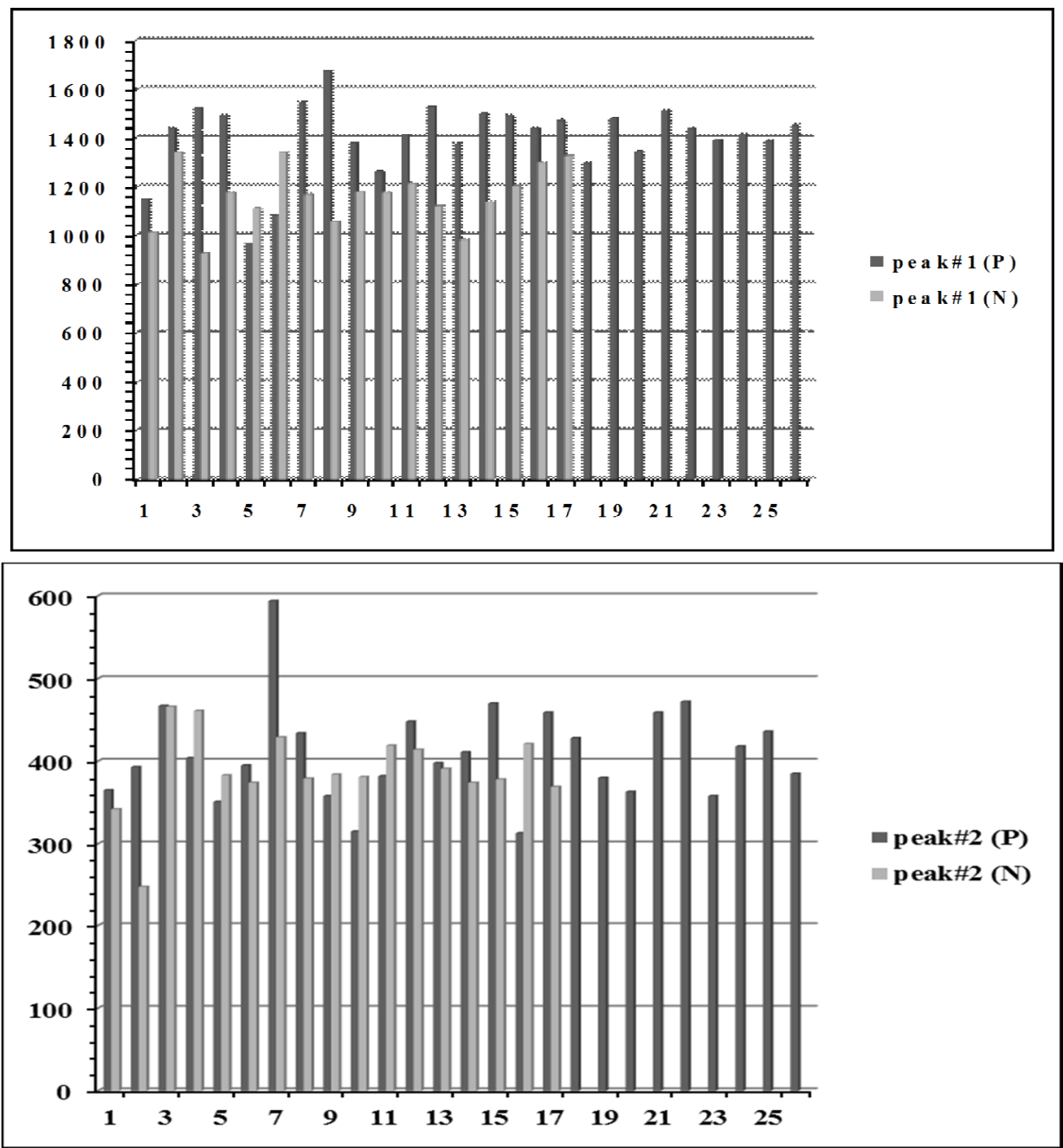

Figure 3. Comparison charts of normal $(N)$ and breast cancer patients $(P)$ for $1^{\text {st }}$ peak $(a)$ and $2^{\text {nd }}$ peak $(\mathbf{b})$.

Table 1. Comparison of obtained results and their sensitivities of different studied groups among three methods of WAXRD, mammography and pathology.

\begin{tabular}{|c|c|c|c|c|c|c|c|c|}
\hline & $\#$ & \multicolumn{2}{|c|}{ WAXRD } & \multicolumn{2}{c|}{ Mammography } & \multicolumn{2}{c|}{ Pathology } & \multirow{2}{*}{ Sensitivity } \\
\hline & & $\mathrm{P}^{*}$ & $\mathrm{~N} *$ & $\mathrm{P}$ & $\mathrm{N}$ & $\mathrm{P}$ & $\mathrm{N}$ & \\
\hline Healthy & 14 & 2 & 12 & - & 14 & - & 14 & 85.7 \\
\hline Suspicious & 12 & 9 & 3 & 7 & 5 & 9 & 3 & 58.3 \\
\hline Cancer & 17 & 17 & - & 17 & - & 17 & - & 100 \\
\hline Total & 43 & & & & & & & \\
\hline
\end{tabular}

Note: *Patient, ${ }^{* *}$ Normal

\section{DISCUSSION}

This study was performed to evaluate the efficiency of WAXRD in detection of breast cancer as a safe and non-invasive method. In addition, comparing the results of pathology as gold standard and mammography in detecting Int. J. Radiat. Res., Vol. 14 No. 2, April 2016 breast cancer with changes in hair structure using WAXRD method were considered.

In this study, the average age of 43 women was $51.3 \pm 11.5$, which is less than screening populations (16). Since in mammography there is radiation hazards to patients, and the sensitivity of 
mammography is decreases with increase of age (16), the goal of this study was to determine if differences between XRD curve of healthy women and breast cancer patients can lead to early detection of breast cancer or prediction of disease without radiation hazards or not.

In this research, the difference between XRD curve of healthy and cancer patients were studied and compared. As figure 1 showed, there are two separate and distinguishable peaks in $9.8 \AA$ and $4.4 \AA$ which reflects d-spacing of hair structure. These data were compatible with those obtained by previous study using synchrotron source which, rings related to d-spacing of hair structure have been reported (9-13). But, as shown in figure 3 , there was an additional ring reflects $4.7 \mathrm{~nm}$ d-spacing in cancer patients $(12,14)$. The $9.8 \AA$ reflection dspacing refers to structure pattern of the alphahelix diameter and the $4.4 \AA$ reflection is referring to the periodic distance of the 3.6alpha-helix structure (18).

As it is shown in figure 3, the overall height of $1^{\text {st }}$ and also $2^{\text {nd }}$ peak in patient group is higher than that of normal women and difference in the height means of two group for $1^{\text {st }}$ peak is significant $(\mathrm{P}<0.05)$. Of the first 17 bars of patient related bars in figure 3-a, 15 of them has greater height in comparison with healthy individuals. Hence, it can be assumed that presence of disease can make differences in hair structure, especially the molecular structure of alpha-helix (18). For mean of $2^{\text {nd }}$ peak height in the two examined groups, the difference was not significant $(\mathrm{P}<0.05)$.

The results in table 1 indicated that WAXRD could provide a proper diagnostic test for patient with breast cancer. In addition, in two groups of suspicious and cancer, the results were completely the same as results of WAXRD and pathology reports. In cancer group, the results were the same among all three studied methods. In comparison between results of WAXRD and pathology, the detection rate of breast cancer by WAXRD was $60.4 \%$. Also, sensitivity of approximately $86 \%$ was achieved. The results obtained here were more accurate than those reported by Corino et al. (64\%) (6).
When comparing the age of donors with results of the method, there was no correlation between age and average height $1^{\text {st }}$ peak of WAXRD. This result is against the results which reported by Corino et al. (17). It can be duo to less number of samples that were tested in this work.

Comparing with mammography, the total procedure time, includes sample preparation; XRD examination time and the curve interpretation, were taking about 48 hours which is longer than mammography. From expense point of view, it was clear that the price of XRD procedure is half of that for mammography. As it is shown in table 1 , the sensitivity of XRD (86\%) was higher than that of mammography (64\%).

Therefore, identification of breast cancer at an early stage, using XRD technique may be provided an alternative early low-cost, without radiation hazards, non-invasive and more sensitive method. Ultimately, there are look promising in detecting breast cancer by XRD method.

Of course, further study should be done to examine more hair samples of breast cancer patients. In addition, the need to provide more experiments is of utmost importance. In conclusion, the findings of this work may be open a new era to investigate whether WAXRD or other modalities such as mammography and also synchrotron based methods are useful to diagnosis of early stages of breast cancer patients.

\section{ACKNOWLEDGMENT}

This work is a part of PhD thesis which financially supported by Isfahan University of Medical Sciences (MUI) (Grant No. 391456).

The authors would like to acknowledge the Radiation Oncology Department of Cancer Institute, Tehran University of Medical Sciences for supporting this research.

Conflict of Interest: Declared none. 


\section{REFERENCES}

1. WHO World cancer report 2012

2. Shahbazi-Gahrouei D (2003) Possible effect of background radiation on cancer incidence in Chaharmahal and Bakhtiari province. Iran J Radiat Res, 1(3): 171-174.

3. Sasieni PD, Shelton J, Ormiston-Smith N, Thomson CS, Silcocks PB (2011) What is the lifetime risk of developing cancer? The effect of adjusting for multiple primaries. Br J Cancer, 105(3): 460-5.

4. Hagen Al, Kvistad KA, Maehle L, Holmen MM, Aase H, Styr $B$, et al. (2007) Sensitivity of MRI versus conventional screening in the diagnosis of BRCA-associated breast cancer in a national prospective series. The Breast, 16(4): 367-74.

5. Carney PA, Miglioretti DL, Yankaskas BC, Kerlikowske K, Rosenberg R, Rutter CM, et al. (2003) Individual and combined effects of age, breast density, and hormone replacement therapy use on the accuracy of screening mammography. Annals of Internal Medicine, 138(3): 168-75.

6. Théberge I, Chang SL, Vandal N, Daigle JM, Guertin MH, Pelletier E, Brisson J (2014) Radiologist interpretive volume and breast cancer screening accuracy in a Canadian organized screening program. J Natl Cancer Inst, 106(3): djt461.

7. Rawashdeh MA, Lee WB, Bourne RM, et al. (2013) Markers of good performance in mammography depend on number of annual readings. Radiology, 269(1): 61-7.

8. Maziar Asghar, Shahbazi-Gahrouei D, Tavakoli Mohammad Bagher, Changizi Vahid (2015) Non invasive XRF analysis of human hair for health state determination of breast tissue. Iran J Cancer Prev, 8(6): e3983.

9. Chaparian A, Oghabian MA, Changizi V, Farquharson MJ (2010) the optimization of an energy-dispersive X-ray diffraction system for potential clinical application.
Applied Radiation and Isotopes, 68(12): 2237-45.

10. Kidane G, Speller RD, Royle GJ, Hanby AM (1999) X-ray scatter signatures for normal and neoplastic breast tissues. Phys Med Biol, 44: 1791-1802.

11. James $V$, Kearsley J, Irving $T$, Amemiya $Y$, Cookson $D$ (1999) Using hair to screen for breast cancer. Nature, 398 (6722): 33-4.

12. Saengkaew $P$, Ussawawongaraya $W$, Khaweerat $S$, Rugmai S, Ouajai S, Luengviriya J, et al. (2011) A preliminary X-ray study on human-hair microstructures for a health-state indicator. World Academy of Science, Eng Technol, 59: 1945-9.

13. Corino GL and French PW (2008) Diagnosis of breast cancer by X-ray diffraction of hair. International Journal of Cancer, 122(4): 847-56.

14. Mistry DAH, Haklani J, French PW (2012) Identification of breast cancer associated lipid in scalp hair. Breast Cancer: Basic and Clinical Research, 6: 113-123.

15. International Atomic Energy Agency (IAEA) TECDOC-950 (1997) Sampling, storage and sample preparation procedures for $\mathrm{X}$-ray fluorescence analysis of environmental materials.

16. Gholizadeh N, Kabiri Z, Kakuee O, Saleh-Kotahi M, Changizi V, Fathollahi V, et al. (2013) Feasibility of breast cancer screening by PIXE analysis of hair. Biological Trace Element Research, 153 (1-3): 105-10.

17. Corino GL, French PW, Lee M, Ajaj MM, Haklani J, Mistry DA, et al. (2009) Characterization of a Test for Invasive Breast Cancer Using X-ray Diffraction of Hair-Results of a Clinical Trial. Breast Cancer: Basic and Clinical Research, 3: 83-90.

18. Pauling L, Corey RB, Branson HR (1951) The structure of proteins; two hydrogen-bonded helical configurations of the polypeptide chain. Proceedings of the National Academy of Sciences of the United States of America, 37(4): 205-11. 